14.1

\title{
Поляризованная флуоресценция в молекулах индола при двухфотонном возбуждении фемтосекундными лазерными импульсами
}

\author{
(c) М.Э. Сасин, ${ }^{1}$ В.И. Тушканов, ${ }^{1,2}$ А.Г. Смолин, ${ }^{1}$ П.С. Штернин, ${ }^{1}$ О.С. Васютинский ${ }^{1}$ \\ ${ }^{1}$ Физико-технический институт им. А.Ф. Иофрер РАН, \\ 194021 Санкт-Петербург, Россия \\ ${ }^{2}$ Санкт-Петербургский академический университет, \\ 194021 Санкт-Петербург, Россия \\ e-mail: sasin@ffm.ioffe.ru
}

(Поступило в Редакцию 23 января 2018 г.)

Исследовано затухание флуоресценции раствора индола в пропиленгликоле при двухфотонном возбуждении линейно и циркулярно поляризованными фемтосекундными лазерными импульсами в диапазоне длин волн 475-510 nm. Определены спектральные зависимости интенсивности флуоресценции $I_{0}$, коэффициента анизотропии $r$ и параметра $\Omega$. Обнаружены и теоретически интерпретированы зависимости этих параметров от энергии возбуждения, в частности, немонотонное изменение интенсивности $I_{0}$, указывающее на рост плотности возбужденных состояний в области энергий выше $5.1 \mathrm{eV}$ и смена знака анизотропии, обусловленная изменением симметрии электронно-колебательных состояний.

DOI: 10.21883/JTF.2018.09.46417.44-18

\section{Введение}

Изучение молекулярной флуоресценции при двухфотонном возбуждении уже в течение многих лет является одним из важнейших инструментов исследования структуры и динамики возбужденных состояний многоатомных молекул красителей в газовой и конденсированной фазах, а также биологических структур [1]. Существенные преимущества метода двухфотонного возбуждения по сравнению с методом однофотонного возбуждения для целей медицины и биологии обусловлены его высоким пространственным разрешением, а также возможностью более глубокого проникновения излучения в биологические ткани. В настоящее время широкое использование в этих исследованиях фемтосекундных импульсных лазеров позволяет реализовывать практически невозмущающие методы исследования структуры и динамики аминокислот, энзимов, белков и других биомолекул $[1,2]$. Основы теории метода двухфотонного возбуждения флуоресценции были заложены в работах Мак Клайна, Каллиса и др. [3-6], которыми было показано, что поляризация флуоресценции может быть описана набором молекулярных параметров, которые в условиях изотропного взаимодействия с молекулами растворителя экспоненциально затухают во времени пропорционально характерным временам жизни возбужденных состояний $\tau_{f}$ и временам вращательной диффузии $\tau_{\text {rot }}$, описывающим изменения пространственного распределения осей возбужденных молекул. Таким образом, появляется возможность использования молекул-зондов, встроенных в большие биомолекулы, для исследования их структуры и динамики в различных условиях, в том числе в составе живых клеток.
В предыдущих исследованиях нашей группы была развита последовательная квантово-механическая теория молекулярной флуоресценции, возникающей при двухцветном двухфотонном (2С2P) возбуждении многоатомных молекул, основанная на методе сферических тензоров [7,8]. Ранее этот метод был успешно применен нами для решения целого ряда задач молекулярной динамики, связанных с описанием векторных корреляций в процессах фотодиссоциации [9-13] и неупругих столкновений [14] молекул. В рамках этой теории были получены общие выражения для временной зависимости интенсивности флуоресценции в произвольных молекулах типа ассиметричного волчка, в которых часть, зависящая от поляризации всех трех фотонов, участвующих в фотопроцессе, была выделена из части, описывающей динамику возбужденных состояний молекул, что позволило резко упростить анализ и разработать новый метод извлечения молекулярных параметров из экспериментальных данных. Этот метод был применен для экспериментального исследования процессов, возникающих при двухфотонном возбуждении целого ряда важных для практики молекул: $n$-терфинил, DMQ (2-метил-5-тетрабутил- $n$-квадрофенил), индол, $\mathrm{NADH}$ (никотинамид-аденин-динуклеотид) [8,15-18].

Настоящая работа посвящена исследованию оптических свойств молекул индола $\left(\mathrm{C}_{8} \mathrm{H}_{7} \mathrm{~N}\right)$ в пропиленгликоле с помощью наблюдения поляризованной флуоресценции при двухфотонном возбуждении импульсным линейно и циркулярно поляризованным излучением фемтосекундного лазера в диапазоне длин волн 475-510 nm. Индол является естественным хромофором аминокислоты триптофан, в значительной мере ответственной за флуоресценцию белков в УФ диапазоне спектра [2]. 
Вследствие большой практической важности аминокислоты триптофан, как структурной части большинства белков и для применения ее в качестве молекулярного зонда, исследованиям динамики возбужденных состояний индола посвящена обширная литература (см., например, [16,19-22]). Вместе с тем в подавляющем числе проведенных до настоящего времени исследований использовались сравнительно низкие энергии возбуждения $4.3-4.7 \mathrm{eV}$, эквивалентные диапазону эффективных длин волн возбуждения 265-295 nm.

В настоящей работе для возбуждения было использовано более коротковолновое излучение, приводящее к заселению высоковозбужденных колебательных состояний двух первых возбужденных электронных уровней энергии молекулы индола ${ }^{1} L_{a}$ и ${ }^{1} L_{b}$ и нижних колебательных состояний третьего возбужденного уровня энергии. Посредством анализа поляризации излучения флуоресценции в работе подробно исследованы полное поглощение и анизотропия двухфотонного заселения электронно-колебательных состояний индола в зависимости от энергии и поляризации возбуждающих фотонов и показано, что в исследованном диапазоне возбуждения анизотропия меняет свой знак. Определены время жизни возбужденных состояний и время вращательной корреляции. Интерпретация полученных результатов осуществлялась на основе квантово-химических расчетов, проведенных из первых принципов, и анализа выражений для поляризации флуоресценции, полученных авторами ранее. В результате построена модель процессов релаксации возбужденных электронноколебательных состояний индола и показано, что наблюдающееся поведение полного сигнала поглощения и его анизотропии, как функция энергии возбуждения, может быть удовлетворительно описано в рамках разработанной авторами теории, использованной в приближении Борна-Оппенгеймера. Определенные в работе время жизни возбужденного состояния $\tau_{f}$ и время вращательной корреляции $\tau_{\text {rot }}$ находятся в хорошем согласии с результатами предыдущих работ $[16,17]$ и работ других авторов.

\section{Экспериментальная методика}

В работе использовались методика проведения экспериментов и обработки полученных экспериментальных результатов, развитые в наших предыдущих работах $[7,8]$. Флуоресценция индола в растворе пропиленгликоля возбуждалась импульсным лазерным излучением, распространявшемся вдоль оси $X$ (рис. 1) и сфокусированным внутри кюветы с раствором. Средняя мощность лазерного излучения на входе в кювету составляла $30 \mathrm{~mW}$ при частоте следования импульсов $80.7 \mathrm{MHz}$ и длительности импульса около $150 \mathrm{fs}$. Излучение имело либо линейную поляризацию, направленную вдоль одной из ортогональных осей $Y$ или $Z$, либо циркулярную

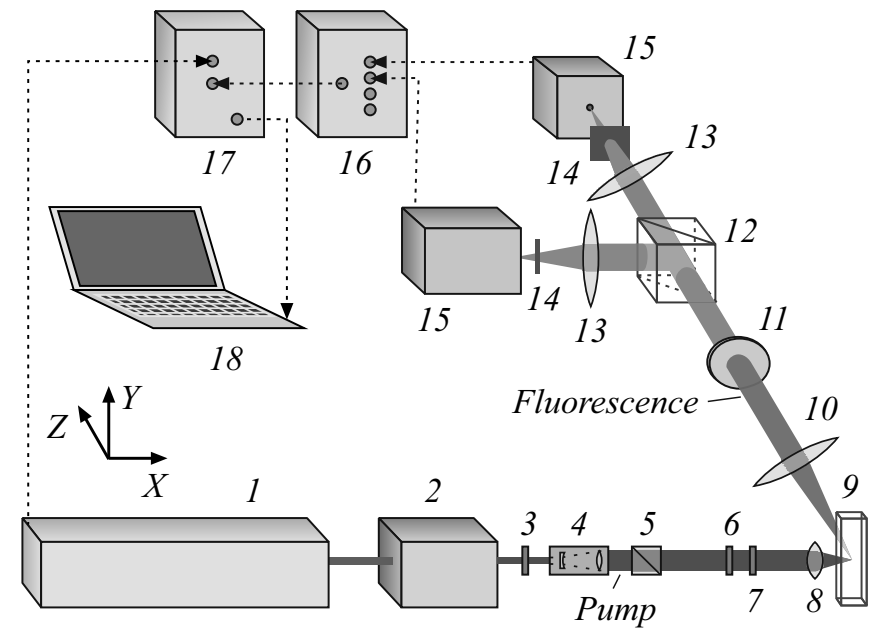

Рис. 1. Схема экспериментальной установки: $1-$ фемтосекундный лазер, 2 - удвоитель частоты, 3 - фазовая пластина $\lambda / 2,4-$ телескопический расширитель, $5-$ поляризационная призма, $6-$ фазовая пластина $\lambda / 2,7-$ фазовая пластина $\lambda / 4,8-$ фокусирующая линза, $9-$ кювета с раствором, $10-$ собирающая линза, $11-$ узкополосный фильтр, 12 - призма Глана, 13 - фокусирующие линзы, $14-$ УФ фильтры, 15 - фотодетекторы, 16 - роутер, 17 - времякорреляционный анализатор, 18 - персональный компьютер. Пунктиром обозначены сигнальные линии.

поляризацию, а степень поляризации для всех конфигураций превышала 99.5\%. Интенсивность двух компонент флуоресценции, линейно поляризованных вдоль взаимно ортогональных осей $X$ и $Y$, детектировались двумя независимыми фотодетекторами, сигналы с которых регистрировались системой время-корреляционного анализа и затем обрабатывались с помощью персонального компьютера. Длина волны возбуждающего излучения изменялась в диапазоне 475-510 nm, что позволяло получать зависимость параметров излучения флуоресценции от энергии возбуждения.

Схема экспериментальной установки приведена на рис. 1. В качестве источника возбуждающего излучения использовался комплекс из импульсного перестраиваемого фемтосекундного Тi:Sa-лазера Mai Tai HP DS (Spectra-Physics) и удвоителя частоты Inspire Blue (Spectra-Physics). После удвоения частоты лазерный пучок проходил через фазовую пластину $\lambda / 2$, телескопический расширитель и поляризационную призмy 10GL08 (Newport), которые использовались для расширения и коллимирования пучка до $4 \mathrm{~mm}$ и его ослабления до заданного значения средней мощности. Средняя мощность лазерного излучения контролировалась измерителем Nova (OPHIR). Затем лазерный пучок проходил через фазовые пластинки $\lambda / 2$ и $\lambda / 4$, с помощью которых осуществлялся контроль направления его линейной поляризации или состояние его циркулярной поляризации. Сформированный таким образом лазерный пучок фокусировался линзой (NA 0.3) в центр кварцевой 
кюветы, содержавшей раствор индола в пропиленгликоле с концентрацией $0.05 \mathrm{~mol} / \mathrm{l}$.

Излучение флуоресценции молекул индола собиралось с помощью линзы и трансформировалось в квазипараллельный пучок, направленный вдоль оси $Z$ и пропускавшийся через узкополосный интерференционный фильтр FF01-390/SP (Semrock) с полосой пропускания $320-370 \mathrm{~nm}$, для отсекания рассеянного лазерного излучения. После прохождения интерференционного фильтра излучение разделялось с помощью призмы Глана на две компоненты со взаимно перпендикулярными поляризациями, параллельными осям $X$ и $Y$. Каждая из этих компонент пропускалась через абсорбционный фильтр УФС5 и фокусировалась на входных отверстиях быстродействующих фотодетекторов на основе лавинных фотодиодов PDM PD-050-CTC (MPD) с полушириной однофотонного отклика $200 \mathrm{ps}$, работавших в режиме счета фотонов. Сигналы от фотодетекторов коммутировались роутером PHR 800 (PicoQuant) и поступали на времякорреляционный анализатор PicoHarp 300 (PicoQuant) с частотой дискретизации $4 \mathrm{ps}$, данные с которого поступали на персональный компьютер для дальнейшей обработки.

\section{Экспериментальные результаты и их обработка}

В ходе эксперимента были получены зависимости затухания интенсивности для двух ортогонально поляризованных компонент флуоресценции молекул индола при разных условиях двухфотонного возбуждения. Условия возбуждения менялись за счет изменения длины волны лазерного излучения в диапазоне $475-510 \mathrm{~nm}$ и за счет изменения типа поляризации. Таким образом, для каждой длины волны возбуждения было зарегистрировано три пары интенсивностей флуоресценции, две из которых соответствовали линейной поляризации возбуждающего излучения вдоль осей $Y$ и $Z$ и третья циркулярной поляризации $L$.

Поскольку для случая линейной поляризации возбуждающего излучения вдоль оси $Z$ излучение флуоресценции не обладало анизотропией при использовавшейся конфигурации детектирования, эти данные применялись лишь для определения коэффициента $G$, учитывающего различие в чувствительности $x$ - и $y$-каналов детектирования поляризованной флуоресценции. Кроме того, поскольку правая и левая циркулярные поляризации излучения приводили к идентичным результатам по флуоресценции, применялась только одна из них левоциркулярная.

На рис. 2 представлены типичные экспериментальные сигналы, полученные при возбуждении молекул индола лазерным излучением, поляризованным вдоль оси $Y$ и циркулярно поляризованным, на длине волны $510 \mathrm{~nm}$. Сигналы флуоресценции с поляризациями вдоль осей $Y, X$ обозначены как 1,2 и 3,4 для линейной и

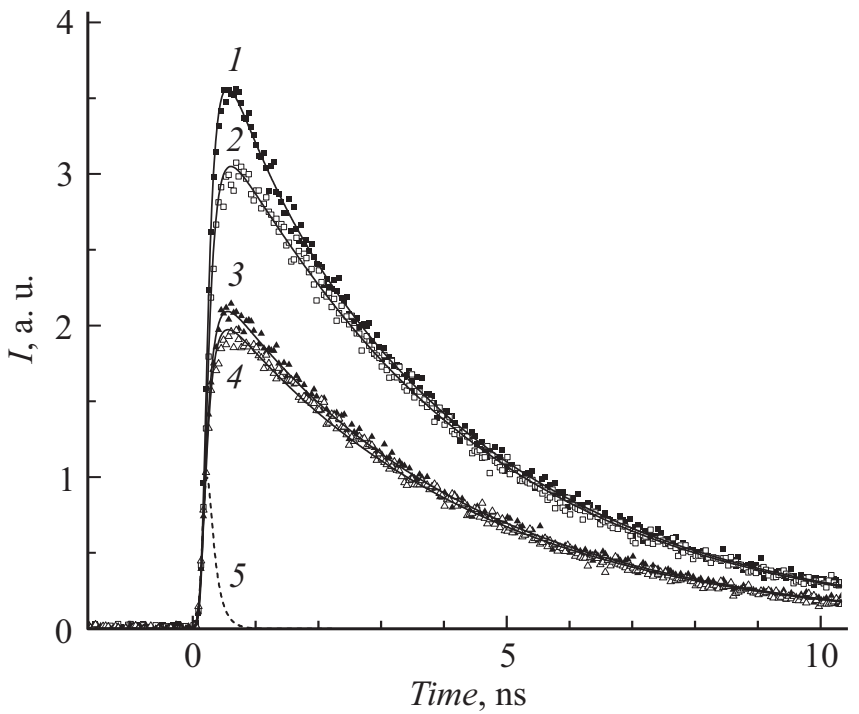

Рис. 2. Сигналы затухания флуоресценции при возбуждении линейно и циркулярно поляризованным излучением с длиной волны $510 \mathrm{~nm}$. Символы - экспериментальные данные, сплошные линии - результаты теоретической подгонки (фитинга), $1-4$ - интенсивность компонент флуоресценции $I_{Y Y y}, I_{Y Y x}$, $I_{L L y}, I_{L L x}$ соответственно, 5 - инструментальная функция отклика детектора $\operatorname{IRF}(t), t=0-$ момент лазерного импульса.

циркулярной поляризации возбуждения соответственно. Общий символ интенсивности сигнала флуоресценции на рис. 2 обозначен как $I_{\mathrm{eef}}$, где индексы $e e=Y Y$, $X X, L L$ обозначают состояние поляризации первых двух (возбуждающих) фотонов, а индекс $f=x, y$ - состояние поляризации фотона флуоресценции. Символами на рис. 2 обозначены экспериментальные результаты, а сплошными кривыми - результаты подгонки по формулам (3)-(11). Разница в интенсивности флуоресценции при различных поляризациях возбуждающего лазерного излучения объясняется различной эффективностью двухфотонного возбуждения для этих поляризаций. Различие интенсивностей компонент сигналов флуоресценции, поляризованных вдоль осей $Y$ и $X$ при одной и той же поляризации возбуждения, обусловлено анизотропным распределением осей возбужденных молекул индола $[1,18]$. Полученные экспериментальные данные содержат изотропную и анизотропную составляющие, по-разному зависящие от времени. Изотропная составляющая интенсивности для случая линейно поляризованного возбуждения может быть представлена в виде $[1,18]$

$$
I_{l}(t)=\frac{1}{3}\left[I_{Y Y y}(t)+2 I_{Y Y x}(t)\right]
$$

Анизотропная составляющая выражается через анизотропию флуоресценции

$$
r_{l}(t)=\frac{I_{Y Y y}(t)-I_{Y Y x}(t)}{I_{Y Y y}(t)+2 I_{Y Y X}(t)} .
$$




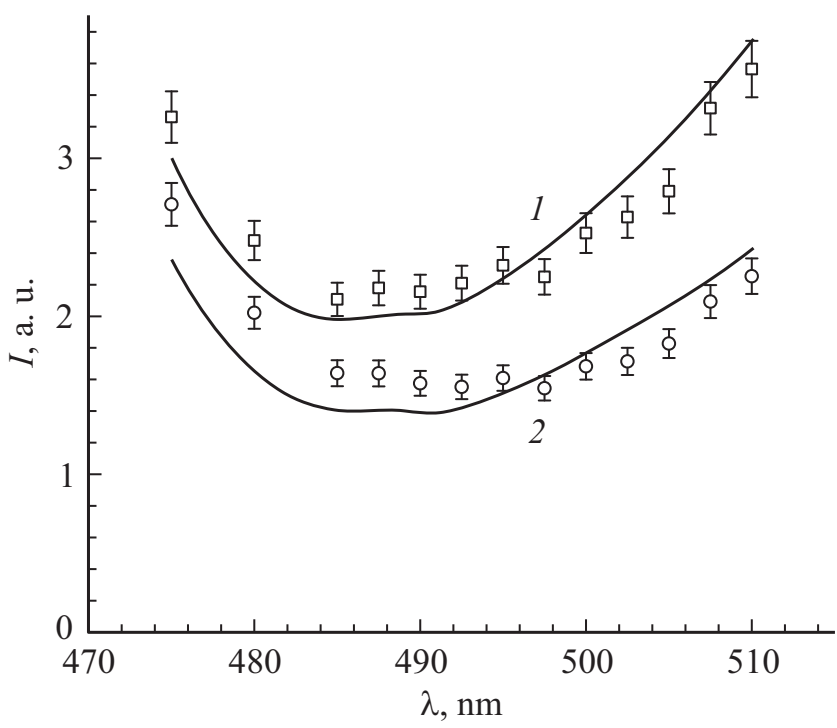

Рис. 3. Зависимость изотропной составляющей интенсивности флуоресценции от длины волны для линейно $I_{l}(1)$ и циркулярно $I_{c}(2)$ поляризованного лазерного излучения. Символы экспериментальные данные, сплошные кривые - результаты подгонки.

В условиях наших экспериментов обе величины в выражениях (1) и (2) затухали по одноэкспоненциальному закону с разными характерными временами. Для изотропной составляющей этим характерным временем являлось $\tau_{f}-$ время жизни нижнего возбужденного состояния молекулы индола, а для анизотропной составляющей - время вращательной корреляции $\tau_{\text {rot }}$, описывающее изменение анизотропии распределения осей возбужденных молекул индола вследствие взаимодействия с молекулами растворителя.

Отметим, что из-за различия в чувствительности $x$ и $y$-каналов регистрации в выражениях (1), (2) необходимо было учитывать коэффициент $G$, являющийся отношением сигналов обоих каналов при отсутствии анизотропии. Для вычисления коэффициента $G$ использовалось отношение интегралов по диапазону измерений:

$$
G=\frac{\int I_{Z Z y} d t}{\int I_{Z Z x} d t}
$$

где интегрирование применялось с целью уменьшения влияния шумов.

Исходя из выражений (1) и (2) и с учетом (3) поляризационные компоненты флуоресценции, полученные в эксперименте, могут быть описаны выражениями:

$$
\begin{gathered}
I_{Y Y y}(t)=G I_{l}(t)\left[1+2 r_{l}(t)\right], \\
I_{Y Y x}(t)=I_{l}(t)\left[1-r_{l}(t)\right] .
\end{gathered}
$$

Полученные экспериментально данные обрабатывались по методике, сходной с методикой, описанной в $[8,16]$. При этом учитывалась инструментальная функция отклика детектора $I R F(t)$, экспериментально определенная на длине волны флуоресценции и показанная на рис. 2 штриховой линией.

Выражения, использованные для аппроксимации экспериментальных данных, представляли собой свертку выражений (4) и (5) с инструментальной функцией $\operatorname{IRF}(t)$ и имели следующий вид:

$$
\begin{gathered}
I_{Y Y y}(t)=G \int_{-\infty}^{t} \operatorname{IRF}\left(t^{\prime}\right) I_{l} e^{\frac{t-t^{\prime}}{\tau_{f}}}\left[1+2 r_{l} e^{\frac{t-t^{\prime}}{\tau_{\mathrm{rot}}}}\right] d t^{\prime}, \\
I_{Y Y x}(t)=\int_{-\infty}^{t} \operatorname{IRF}\left(t^{\prime}\right) I_{l} e^{\frac{t-t^{\prime}}{\tau_{f}}}\left[1-r_{l} e^{\frac{t-t^{\prime}}{\tau_{\mathrm{rot}}}}\right] d t^{\prime},
\end{gathered}
$$

где $I_{l}$ и $r_{l}$ - не зависящие от времени максимальные значения изотропной составляющей интенсивности и анизотропии для случая линейно поляризованного возбуждения.

Аппроксимация выполнялась в два этапа. На первом этапе на основе выражений (1), (6) и (7) с помощью процедуры подгонки определялись время жизни возбужденного состояния $\tau_{f}$ и интенсивность $I_{l}$. На втором этапе полученные ранее параметры $\tau_{f}$ и $I_{l}$ использовались в качестве констант и на основании выражений (2), (6), (7) определялись величины $\tau_{\text {rot }}$ и $r$. Для случая циркулярно поляризованного возбуждения индексы $x$ и $y$ в выражениях (1), (2), (4)-(7) должны быть поменяны местами, а выражения $I_{l}$ и $r_{l}$ заменялись на $I_{c}$ и $r_{c}$ соответственно.

Результаты аппроксимации показаны на рис. 2 сплошными кривыми. С помощью описанной методики были получены наборы параметров для всех длин волн и типов поляризации возбуждающего лазерного излучения.

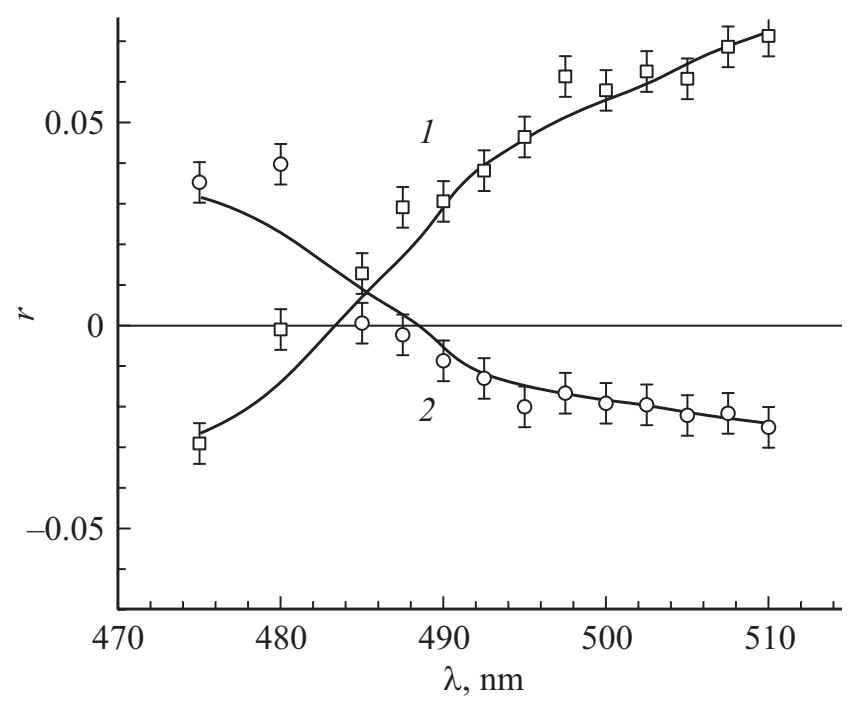

Рис. 4. Зависимость анизотропии флуоресценции от длины волны для линейно $I_{l}(1)$ и циркулярно $I_{c}(2)$ поляризованного лазерного излучения. Символы - экспериментальные данные, сплошные кривые - результаты подгонки. 


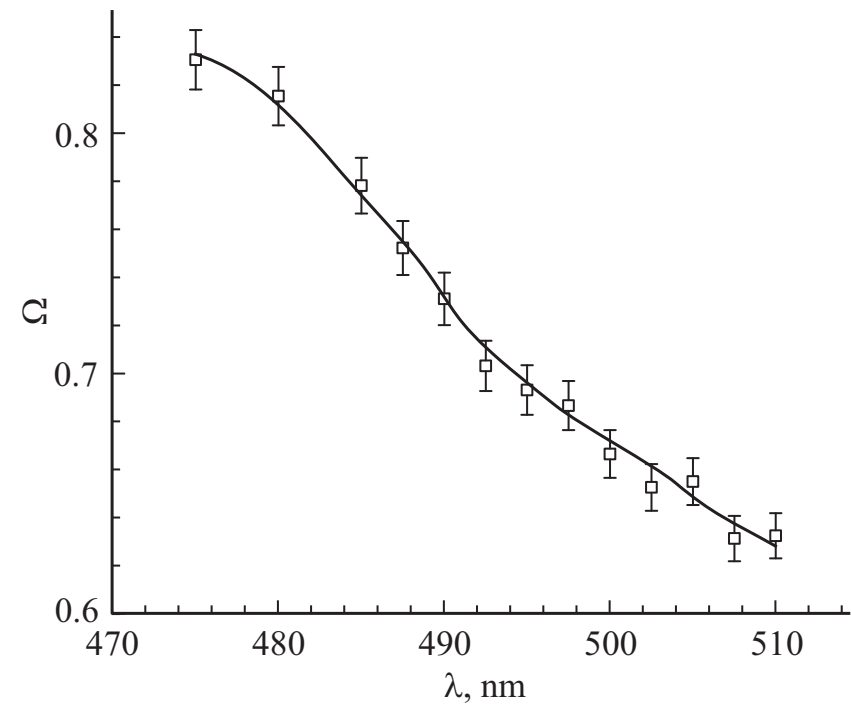

Рис. 5. Зависимость параметра $\Omega$ от длины волны лазерного излучения. Символы - экспериментальные данные, сплошная кривая - результаты подгонки.

В частности, были определены значения характерных времен $\tau_{f}=3.9 \pm 0.02 \mathrm{~ns}$ и $\tau_{\text {rot }}=0.86 \pm 0.07 \mathrm{ns,} \mathrm{кото-}$ рые не зависели от длины волны возбуждающего излучения и находились в хорошем согласии с результатами, полученными в наших предыдущих работах $[16,17]$.

Значения изотропных составляющих интенсивности для линейно $I_{l}$ и циркулярно $I_{c}$ поляризованного излучения лазера, как функция его длины волны, приведены на рис. 3. Как видно, обе величины изменяются в общем подобным образом, имея максимумы на границах исследованного диапазона длин волн и минимум в области 480-500 nm.

Зависимость параметров анизотропии $r_{l}, r_{c}$ от длины волны возбуждения приведены на рис. 4. Как видно, параметры анизотропии для случаев линейно и циркулярно поляризованного возбуждения изменяются в противофазе друг к другу и меняют знак приблизительно около $482-484 \mathrm{~nm}$.

На рис. 5 приведена зависимость известного коэффициента $\Omega$, характеризующего симметрию двухфотонного перехода

$$
\Omega=\frac{I_{L L}}{I_{Y Y}}
$$

от длины волны лазерного излучения для линейной и циркулярной поляризаций. Как видно из рис. 5, в исследованном спектральном диапазоне этот коэффициент везде положителен и монотонно возрастает при уменьшении длины волны возбуждения, однако не достигая критического значения $\Omega=3 / 2$, указывающего на присутствие неполносимметричного возбужденного состояния [1].
Таблица 1. Энергии нижних возбужденных электронных состояний и дипольные моменты перехода при возбуждении из основного состояния молекулы индола в пропиленгликоле

\begin{tabular}{c|c|c|c|r|c}
\hline \multirow{2}{*}{ Состояние } & \multirow{2}{*}{ Симметрия } & \multirow{2}{*}{ Энергия } & \multicolumn{3}{|c}{$\begin{array}{c}\text { Дипольный момент } \\
\text { перехода, а.u. }\end{array}$} \\
\cline { 4 - 6 } & & & $X^{\prime}$ & $Y^{\prime}$ & $Z^{\prime}$ \\
\hline 1 & $A^{\prime}$ & 4.32 & 0.5151 & -1.1421 & 0.0 \\
2 & $A^{\prime}$ & 4.56 & 0.8966 & -0.0290 & 0.0 \\
3 & $A^{\prime}$ & 5.43 & 2.7162 & -0.2314 & 0.0 \\
4 & $A^{\prime}$ & 5.91 & -0.8777 & 0.9732 & 0.0
\end{tabular}

\section{Теоретическая интерпретация и обсуждение полученных результатов}

Для интерпретации полученных экспериментальных данных были использованы результаты квантовомеханических расчетов электронной структуры индола в растворе пропиленгликоле, проведенных в нашей предыдущей работе [17]. Часть этих результатов представлена в табл. 1 .

Первые два электронные состояния молекулы индола в табл. 1 широко известны и обычно обозначаются ${ }^{1} L_{a}$ и ${ }^{1} L_{b}$ [1]. Минимальные энергии возбуждения этих уровней $T_{e}$ из основного электронного состояния приведены в первых двух строчках табл. 1 и равны 4.32 и $4.56 \mathrm{eV}$, а дипольные моменты однофотонных переходов в эти электронные состояния из основного состояния, приведенные в столбцах 4-6, лежат в плоскости молекулы индола и практически перпендикулярны друг другу. Использованный в настоящей работе интервал энергий двухфотонного возбуждения лежал существенно выше этих значений, но ниже энергии следующего электронного состояния $T_{e}=5.43 \mathrm{eV}$, приведенной в третьей строчке табл. 1. Таким образом, экспериментальные сигналы, приведенные на рис. 3-5, в значительной мере обусловлены заселением высоковозбужденных колебательно-вращательных уровней энергии электронных состояний ${ }^{1} L_{a}$ и ${ }^{1} L_{b}$. Вместе с тем проведенный анализ показал, что в условиях наших экспериментов существенный вклад в коротковолновую часть сигналов на рис. 3-5 вносило заселение третьего возбужденного электронного состояния в табл. 1, которое оказалось возможным вследствие значительного уширения линии возбуждения использованного импульсного лазера, которое составляло около $4 \mathrm{~nm}$.

Интерпретация экспериментальных сигналов, приведенных на рис. 3-5, осуществлялась на основе теории, разработанной в наших предыдущих работах [7,8], с использованием данных табл. 1 и с учетом спектров колебательного возбуждения молекул индола и профиля линии использованного фемтосекундного лазера. Расчет колебательных спектров возбуждения для оптических 
Таблица 2. Значения параметров $M_{0}^{e}(2,2), M_{2}^{e}(0,2), M_{2}^{e}(2,2)$, нормированных на $M_{0}^{e}(0,0)$, и параметров $r_{Y Y}, r_{R R}$ для каждого возбужденного электронного состояния

\begin{tabular}{c|c|c|r}
\hline Параметры & $\begin{array}{c}\text { Электронное } \\
\text { состояние 1 }\end{array}$ & $\begin{array}{r}\text { Электронное } \\
\text { состояние 2 }\end{array}$ & $\begin{array}{c}\text { Электронное } \\
\text { состояние 3 }\end{array}$ \\
\hline$M_{0}^{e}(2,2) / M_{0}^{e}(0,0)$ & $0.48 \pm 0.1$ & $2.33 \pm 0.31$ & $1.43 \pm 0.28$ \\
$M_{2}^{e}(0,2) / M_{0}^{e}(0,0)$ & $0.44 \pm 0.12$ & $-0.54 \pm 0.13$ & $-0.073 \pm 0.04$ \\
$M_{2}^{e}(2,2) / M_{0}^{e}(0,0)$ & $0.17 \pm 0.06$ & $-0.11 \pm 0.06$ & $-0.31 \pm 0.16$ \\
$r_{Y Y}$ & $0.15 \pm 0.3$ & $-0.084 \pm 0.02$ & $-0.036 \pm 0.014$ \\
$r_{R R}$ & $-0.058 \pm 0.012$ & $0.0076 \pm 0.02$ & $0.037 \pm 0.016$
\end{tabular}

переходов из основного электронного состояния в каждое исследуемое электронное состояние проводился в приближении Франка-Кондона с учетом заселенности колебательных уровней основного электронного состояния при температуре $300 \mathrm{~K}$. Этот расчет был осуществлен на основе вычислительного пакета GAUSSIAN [23] с параметрами, использованными ранее в работе [17], причем колебательные спектры поглощения определялись с учетом обертонов и составных частот. Полученные колебательные спектры возбуждения имели несколько пиков вблизи нижних границ электронных состояний, а затем плавно затухали по амплитуде с повышением энергии возбуждения. Однако после последующей свертки колебательного спектра возбуждения с профилем линии фемтосекундного лазера с шириной $4 \mathrm{~nm}$, сложная структура колебательного спектра возбуждения становилась практически неразличимой.

Для фитинга экспериментальных данных на рис. 3-5 были использованы теоретические выражения, полученные в наших предыдущих работах $[7,8]$ и основанные на определении из эксперимента набора молекулярных параметров $M_{K}\left(R, R^{\prime}\right)$, причем учет влияния колебательных спектров возбуждения осуществлялся в приближении Борна-Оппенгеймера. Для используемой в экспериментальной геометрии параметры анизотропии $r_{l}, r_{c}$ и $\Omega$ могут быть следующим образом выражены через значения молекулярных параметpoв $[8]$ :

$$
\begin{gathered}
r_{Y Y}=\frac{2}{5} \frac{M_{2}(0,2)+M_{2}(2,2) / \sqrt{7}}{M_{0}(0,0)+2 M_{0}(2,2) / \sqrt{5}}, \\
r_{R R}=-\frac{1}{\sqrt{35}} \frac{M_{2}(2,2)}{M_{0}(2,2)}, \\
\Omega=\frac{3 M_{0}(2,2)}{\sqrt{5} M_{0}(0,0)+2 M_{0}(2,2)} .
\end{gathered}
$$

Молекулярные параметры $M_{K}\left(R, R^{\prime}\right)$, детально описанные в работах $[7,8]$, содержат в себе полную информацию о симметрии двухфотонного возбуждения, задаваемую через тензоры двухфотонного возбуждения $S_{R \gamma}$ и флуоресценции $F$. Необходимый для анализа набор молекулярных параметров может быть сравнительно легко определен из экспериментальных данных. В приближении Борна-Оппенгеймера тензор двухфотонного возбуждения $S_{R \gamma}$ может быть представлен в виде

$$
S_{R \gamma}=S_{R \gamma}^{e}\left\langle Q_{v^{\prime}} \mid Q_{v^{\prime \prime}}\right\rangle+V_{R \gamma}
$$

где матричный элемент в угловых скобках представляет собой интеграл перекрытия колебательных волновых функций $\theta$, а тензор $S_{R \gamma}^{e}$ зависит только от параметров электронных состояний.

Тензор $V_{R \gamma}$ в выражении (12) содержит в себе высшие члены разложения тензора $S_{R \gamma}$ и характеризует колебательно-индуцированные переходы, которыми можно пренебречь для условий данного эксперимента. При этом выражение для молекулярных параметров $M_{K}\left(R, R^{\prime}\right)$ для каждого электронного состояния можно представить в виде

$$
M_{K}\left(R, R^{\prime}\right) \approx M_{K}^{e}\left(R, R^{\prime}\right)\left|\left\langle\theta_{v^{\prime}} \mid \theta_{v^{\prime \prime}}\right\rangle\right|^{2},
$$

где величины $M_{K}^{e}\left(R, R^{\prime}\right)$ зависят только от электронных волновых функций молекулы.

Для учета одновременного возбуждения нескольких электронных состояний осуществлялось суммирование по всем соответствующим молекулярным параметрам. Фитинг экспериментальных данных осуществлялся на основе выражений (2), (8), (9) и (11) методом наименьших квадратов с последовательным определением сначала $M_{0}^{e}(0,0), M_{0}^{e}(2,2)$ параметров через параметр $\Omega$, затем $M_{2}^{e}(2,2)$ через параметр $r_{R R}$, и затем $M_{2}^{e}(0,2)$ через параметр $r_{Y Y}$. Результаты фитинга приведены в табл. 2 и на рис. $3-5$.

Погрешность определения параметров $M_{2}^{e}(0,2)$, $M_{2}^{e}(2,2)$ несколько выше, чем $M_{0}^{e}(0,0), M_{0}^{e}(2,2)$, так как параметры $M_{2}^{e}(0,2), M_{2}^{e}(2,2)$ входят в выражения для разности сигналов флуоресценции. Как видно из рис. 3 , при двухфотоннном возбуждении на длинах волн больше $492 \mathrm{~nm}$ имеются практически прямолинейные участки спектра. Эти участки хорошо аппроксимируются вкладами первых двух возбужденных электронных состояний, причем монотонное уменьшение сигнала флуоресценции в этих областях обусловлено уменьшением заселенности высоковозбужденных колебательных состояний. При длинах волн возбуждения меньше $492 \mathrm{~nm}$ наблюдается увеличение интенсивности двухфотонного поглощения 
за счет вклада нижних колебательных состояний третьего возбужденного электронного состояния. Согласно результатам расчета, приведенным в табл. 1, четвертое возбужденное электронное состояние находится значительно выше и не оказывает влияние в исследуемом спектральном диапазоне. Таким образом, наши экспериментальные результаты и интерпретация позволяют подтвердить наличие третьего электронного состояния при расчетном значении энергии.

Как видно из рис. 4, анизотропия флуоресценции монотонно возрастает при возрастании длины волны в пределах исследованного спектрального интервала при линейно поляризованном возбуждении и монотонно убывает при циркулярно поляризованном возбуждении. При этом изменения анизотропии для случаев линейно и циркулярно поляризованного возбуждений происходят в противофазе и в обоих случаях анизотропия меняет знак приблизительно при $485 \mathrm{~nm}$. Отметим, что существование отличных от нуля значений анизотропии обусловлено анизотропным распределением (выстраиванием) осей возбужденных молекул, которое образуется при двухфотонных переходах под действием поляризованного лазерного излучения $[1,7]$ и находит свое отражение в поляризации наблюдающейся флуоресценции. Монотонное изменение анизотропии при изменении длины волны возбуждения в обоих случаях обусловлено изменением симметрии тензора двухфотонного возбуждения (12) второго ранга, $R=2$, при последовательном заселении первого, второго и третьего возбужденных состояний молекулы индола, как описано выше. При этом противофазное изменение значений параметра анизотропии для линейно и циркулярно поляризованном возбуждении обусловлено разными знаками выстраивания молекулярных осей, реализующихся для этих двух типов возбуждения [24].

Как видно из рис. 5, параметр $\Omega$ (см. выражения (8) и (11)) монотонно убывает в исследованном спектральном диапазоне, как функция длины волны возбуждения, будучи везде положительным, но не достигая критического значения $\Omega=3 / 2$, указывающего на присутствие неполно-симметричного возбужденного состояния [1]. Таким образом, приведенные на рис. 5 результаты подтверждают расчетные данные о симметрии первых трех возбужденных состояний индола, приведенных в табл. 1.

\section{Выводы}

Таким образом, в настоящей работе исследовано затухание поляризованной флуоресценции молекул индола в пропиленгликоле при двухфотонном возбуждении линейно и циркулярно поляризованными фемтосекундными лазерными импульсами в диапазоне длин волн 475-510 nm. Исследованы полное поглощение и анизотропия двухфотонного заселения электронноколебательных состояний индола в зависимости от энергии и поляризации возбуждающих фотонов. Обнару- жено, что анизотропии при линейном и циркулярном возбуждениях в исследованном спектральном диапазоне возбуждения изменяются в противофазе друг другу и меняют свой знак. Определены время жизни возбужденных состояний и время вращательной корреляции. Интерпретация полученных результатов проведена на основе квантово-химических расчетов, проведенных из первых принципов, и анализа выражений для поляризации флуоресценции, полученных авторами ранее. Построена модель процессов релаксации возбужденных электронно-колебательных состояний индола и показано, что наблюдающееся поведение полного сигнала поглощения и его анизотропии, как функция энергии возбуждения, может быть удовлетворительно описана в рамках разработанной авторами теории, использованной в приближении Борна-Оппенгеймера.

Работа выполнена при финансовой поддержке Российского научного фонда (РНФ), проект № 14-13-00266.

\section{Список литературы}

[1] Lakowicz J.R. ed. Topics in Fluorescence Spectroscopy. Vol. 5. Nonlinear and Two-Photon-Induced Fluorescence. NY: Kluwer Academic Publ., 2002.

[2] Demchenko A.P. // Ultr. Spec. Prot. 1986. P. 312.

[3] McClain W.M. // J. Chem. Phys. 1972. Vol. 57. P. 2264.

[4] Wan C., Johnson C.K. // Chem. Phys. 1994. Vol. 179. P. 513.

[5] Callis P.R. // Ann. Rev. Phys. Chem. 1997. Vol. 48. P. 271.

[6] Lakowicz J.R., Gryczynski I., Malak H., Gryczynski Z. /I Photochem. Photobiol. 1996. Vol. 64. P. 632.

[7] Shternin P.S., Gericke K.H., Vasyutinskii O.S. // Mol. Phys. 2010. Vol. 108. P. 813.

[8] Denicke S., Gericke K.-H., Smolin A.G., Shternin P.S., Vasyutinskii O.S. // J. Phys. Chem. A. 2010. Vol. 114. P. 9681.

[9] Lee S.K., Silva R., Thamanna S., Vasyutinskii O.S., Suits A.G. // J. Chem. Phys. 2006. Vol. 125. P. 144318.

[10] Korovin K.O., Picheyev B.V., Vasyutinskii O.S., Valipour H., Zimmermann D. // J. Chem. Phys. 2000. Vol. 112. P. 2059-2062.

[11] Shternin P.S., Vasyutinskii O.S. // J. Chem. Phys. 2008. Vol. 128. P. 194314.

[12] Kuznetsov V.V., Vasyutinskii O.S. // J. Chem. Phys. 2007. Vol. 127. P. 044308.

[13] Kupriyanov D.V., Sevastianov B.N., Vasyutinskii O.S. // Z. für Phys. D. 1990. Vol. 15. P. 105-115.

[14] Balint-Kurti G.G., Vasyutinskii O.S. // J. Phys. Chem. A. 2009. Vol. 113. P. 14281-14290.

[15] Herbrich S., Gericke K.-H., Smolin A.G., Vasyutinskii O.S. // J. Phys. Chem. A. 2014. Vol. 118. P. 5248.

[16] Herbrich S., Al-Hadhuri T., Gericke K.-H., Shternin P.S., Smolin A.G., Vasyutinskii O.S. // J. Chem. Phys. 2015. Vol. 142. P. 024310.

[17] Сасин М.Э., Тушканов В.И., Смолин А.Г., Васютинский О.С. // Опт. и спектр. 2017. Т. 123. № 4. С. 555-560. [Sasin M.E., Tushkanov V.I., Smolin A.G., Vasyutinskii O.S. // Opt. Spectr. 2017. Vol. 123. N 4. P. 569-573.] 
[18] Васютинский О.С., Смолин А.Г., Oswald C., Gericke K.-H. // Опт. и спектр. 2017. Т. 122. № 4. C. 622-626. [Vasyutinskii O.S., Smolin A.G., Oswald C., Gericke K.H. // Opt. Spectr. 2017. Vol. 122. N 4. P. 602-606.]

[19] Albinsson B., Norden B. // J. Phys. Chem. 1992. Vol. 96. P. 6204.

[20] Hager J.W., Wallace S.C. // J. Phys. Chem. 1983. Vol. 87. P. 2121.

[21] Povedailo V.A., Yakovlev D.L. // J. Appl. Spectrosc. 2008. Vol. 75. P. 336.

[22] Kang C., Korter T.M., Pratt D.W. // J. Chem. Phys. 2005. Vol. 122. P. 174301.

[23] Frisch M.J., Trucks G.W., Schlegel H.B. et al. Wallingford. Gaussian Gaussian, Inc., 09. 2009.

[24] Chichinin A.I., Shternin P.S., Gödecke N., Kauczok S., Maul C., Vasyutinskii O.S., Gericke K.-H. // J. Chem. Phys. 2006. Vol. 125. P. 034310. 